\title{
Fragmentation Studies of Noncovalent Sugar-Sugar Complexes by Infrared Atmospheric Pressure MALDI
}

\author{
Christopher E. Von Seggern and Robert J. Cotter \\ Department of Pharmacology and Molecular Sciences, Johns Hopkins University School of Medicine, \\ Baltimore, Maryland, USA
}

\begin{abstract}
An investigation of sugar-sugar noncovalent complex fragmentation was conducted using a $2.94 \mu \mathrm{m}$ Er:YAG laser for infrared (IR) atmospheric pressure matrix-assisted laser desorption / ionization (AP MALDI) on an ion trap mass spectrometer (ITMS). This approach allowed the analysis of weak noncovalent complexes between a variety of biologically relevant oligosaccharides. The strength of interaction varied with different sugar structures, potentially due to varying strength of hydrogen bonding networks. In some cases, fragmentation of intramolecular sugar bonds preceded breakdown of the noncovalent complex. This result appeared primarily when complexes contained sugars with at least one sialic acid. Globotrios dimers also showed intramolecular fragmentation in preference to breakdown of the noncovalent dimer. This technique will allow further study of sugar-sugar interactions known to play a role in cellular interactions. (J Am Soc Mass Spectrom 2003, 14, 1158-1165) (c) 2003 American Society for Mass Spectrometry
\end{abstract}

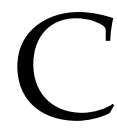
arbohydrates play key roles in cellular interactions involved in the immune system. In particular, sialic acids are the key binding determinants for the siglec protein family. These proteincarbohydrate interactions are vital for cellular adhesion and normal function of the immune system [1-5]. Furthermore, protein-carbohydrate interactions are known to vary in binding affinity due to differing linkages within the carbohydrate. It is clear that sugars themselves have crucial roles in determining specificity of binding, and it has been speculated that carbohydrates are involved in cellular interactions themselves, without the need for protein involvement [6,7]. The wide variety of adoptable structures allows carbohydrates to have a range of potential hydrogen bond interactions with other sugars. Sugars have been demonstrated to be the key sites of interactions between a variety of glycosphingolipids (GSLs) [8]. The sugar portion of the GSL has been shown to orient in a fashion amenable to interactions with other sugars $[6,8]$. These interactions often lead to downstream signaling pathways, involved in a variety of functions crucial for immune function [9]. Currently, interactions between a variety of sugars have been identified, including those between Lewis ${ }^{\mathrm{x}}$ and Lewis ${ }^{\mathrm{x}}$, and other sugars, leading to proper function of the immune system [10, 11].

Published online August 11, 2003

Address reprint requests to Dr. R. J. Cotter, Department of Pharmacology and Molecular Sciences, Johns Hopkins University School of Medicine, 725 N. Wolfe Street, B-7 Biophysics Bldg., Baltimore, MD. E-mail: rcotter@jhmi.edu
Studying these interactions more thoroughly could lead to further discovery of sugar-sugar interactions responsible for downstream effects in the immune system.

Whole cell assays are currently the method of choice for determining sugar binding and specificity [7]. While these techniques have proven useful, they rely upon the multi-valiancy of the cells to determine specificity and binding affinities between different sugars. NMR-based techniques have also been used to determine binding interactions of known carbohydrate clusters [10]. These techniques produce complex results that often require a large amount of time and energy to interpret. New analytical methods need to be established that are rapid, easy to interpret, and rely on individual interactions, in order to study noncovalent complexes involving sugars.

Mass spectrometry has been used to study weak noncovalent interactions between a variety of macromolecules [12-17]. Protein-protein, protein-sugar, protein-DNA, protein-RNA and protein-metal interactions have all been studied using mass spectrometry. Electrospray has been established as the technique of choice to study noncovalent complexes due to its soft ionization and sample conditions near physiological $\mathrm{pH}$ [12-14]. ESI often requires a volatile buffer, which can impede noncovalent complex formation. Also, ESI is not amenable to high throughput analyses, as is possible with MALDI techniques. Matrix-assisted laser desorption/ionization (MALDI) has also be used to study noncovalent interactions [15-17]. However, this technique is limited due to the acidic nature of most MALDI 
matrices, which could disrupt salt bridges. Harsh acid conditions can often break down weak noncovalent interactions. Most MALDI matrices have a $\mathrm{pH}$ lower than the pKa of the acidic side chains of amino acids. Protonation of acidic residues eliminates the potential for salt bridges, and could consequently disrupt noncovalent complexes based on ionic interactions. Liquid samples are difficult to study since most MALDI techniques rely on cocrystallization with matrix and analyte. Liquid samples would be more representative of solution based interactions, which might be perturbed in crystalline form.

Recently, Laiko and coworkers combined an atmospheric pressure MALDI source to an orthogonal acceleration time of flight mass spectrometer [18]. Subsequently, this source was coupled to an ion trap mass spectrometer (ITMS) [19]. AP MALDI produces ions with low internal energy, due to collisions with atmospheric gases following ionization, which may reduce the incidence of metastable fragmentation that can occur by MALDI. This technique is ideally suited for studying weak interactions, since ions or complexes with lower internal energy are less likely to dissociate. Furthermore, AP MALDI does not require vacuum at the source, and may be readily coupled to ion trap mass spectrometers [19-25]. This configuration allows the ease of sample preparation of MALDI with the potential for $\mathrm{MS}^{\mathrm{n}}$ experiments provided by ITMS.

Recently, Laiko and coworkers equipped an ITMS with an infrared (IR) optical parametric oscillator (OPO) laser tuned to $3 \mu \mathrm{m}$ for the analysis of peptides [26]. Our lab subsequently equipped an atmospheric pressure MALDI source with an infrared laser of wavelength $2940 \mathrm{~nm}$ (Bioscope UV+, Bioptic Lasersysteme, Berlin, Germany), for the analysis of carbohydrates [27]. The source was fitted to a commercial ion trap mass spectrometer (ThermoFinnigan, San Jose, CA). The infrared laser wavelength $(2940 \mathrm{~nm})$ coincides with the $\mathrm{O}-\mathrm{H}$ stretching frequency, which allows the use of water and glycerol as a matrix. The atmospheric pressure source is further compatible with liquids and aqueous mixtures, which are rapidly depleted under the normal high vacuum conditions of most MALDI mass spectrometers. Glycerol based samples have a longer lifetime at atmospheric pressure, which provides sufficient time to perform multiple $\mathrm{ms} / \mathrm{ms}$ experiments, in both positive and negative mode. Furthermore, its near physiological $\mathrm{pH}$, additional $\mathrm{O}-\mathrm{H}$ bonds, and high viscosity, makes glycerol ideally suited as a matrix for the study of noncovalent interactions.

IR AP MALDI mass spectrometry is ideal for the study of weak noncovalent interactions. Infrared MALDI has been demonstrated to be softer than UV MALDI, while at the same time AP MALDI is a softer ionization technique than vacuum MALDI. Spectra obtained by MALDI are less complicated than those obtained by ESI, and are more easily interpreted. Finally, MALDI based techniques are amenable to high throughput searches, which would be required to study a large number of noncovalent interactions. The soft ionization can ensure complex survival during the ionization process. Liquid matrices can be used to produce near physiological conditions, ensuring proper conditions for noncovalent complex formation. Finally, the ITMS provides the ability to test the strength of the complexes under gas-phase collisions, and provides the opportunity to test the strength of complex formation. In this work we present IR AP MALDI analysis of noncovalent sugar-sugar interactions.

\section{Experimental}

\section{Oligosacchardies}

Disaialyl,monofucosyllacto- $N$-hexaose, disialyl-lacto- $N$ tetraose, difucosyllacto- $N$-hexaose(a), and trifucosyllacto- $N$-hexaose were purchased from Glyko (Novato, CA). Oligomannose-3 was purchased from Glykotech (Rockville, MD). Lewis ${ }^{x}$ and globotriose were purchased from Dextra Laboratories (Reading, UK). All samples were dissolved to $1 \mathrm{mM}$ concentration in 18 $\mathrm{M} \Omega$ MilliPure water.

\section{Liquid AP-MALDI Matrix}

Glycerol was obtained from Sigma (St. Louis, MO) and used without further purification.

\section{Liquid Infrared AP-MALDI/ITMS}

Analyses were performed on a ThermoFinnigan LCQ classic quadrupole ion trap mass spectrometer (San Jose, CA) equipped with a modified Mass Technologies Inc. (Burtonsville, MD) atmospheric pressure MALDI source. The source is currently equipped with a Bioptic Lasersysteme Bioscope UV+ laser (Berlin, Germany) that contains both a Nd:YAG laser (355 nm) and an Er:YAG laser (2940 nm). The Er:YAG laser was utilized for this work. The laser was focused with a sapphire lens $(\mathrm{F}=100 \mathrm{~mm})$. The Er:YAG laser generated approximately $100 \mathrm{~ns}$ pulses at $5 \mathrm{~Hz}$ with approximately 400 $\mu \mathrm{J} /$ pulse. The laser was run asynchronously with the trapping cycle and spectra were acquired at $400 \mathrm{~ms}$ per scan. A potential of $2.5 \mathrm{kV}$ was applied between the AP-MALDI sample plate and the inlet capillary. The capillary temperature was set to $200{ }^{\circ} \mathrm{C}$.

Samples were prepared by spotting $1 \mu \mathrm{L}$ of analyte in approximately $1 \mu \mathrm{L}$ of glycerol. The resulting mixture was then analyzed in liquid form.

\section{Results and Discussion}

Stemming from earlier sugar fragmentation studies [27], it was noted that at high concentrations, many sugars formed noncovalent homodimers (data not shown). Noncovalent mixed dimers could also be formed from two different sugars (Figure 1a). Figure 1a illustrates a typical positive mode IR AP MALDI spec- 


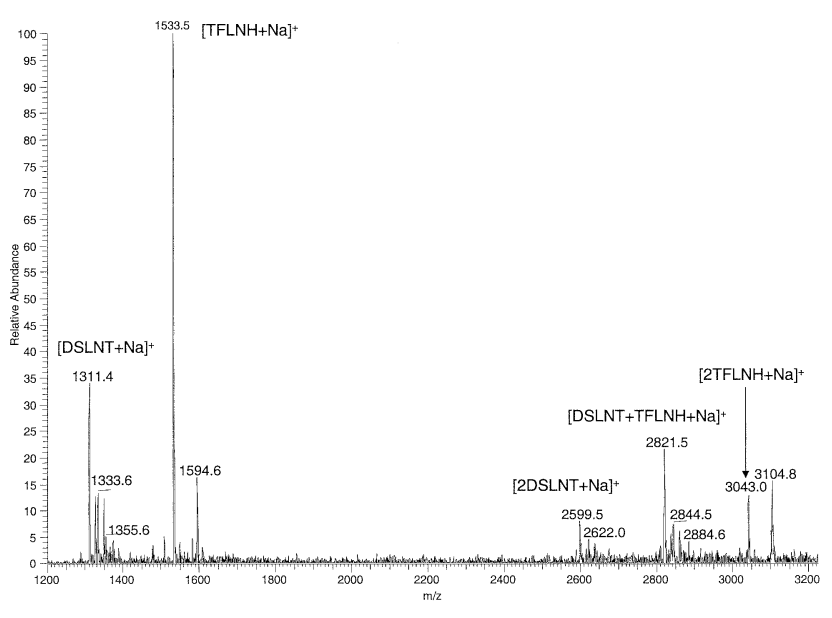

(a)

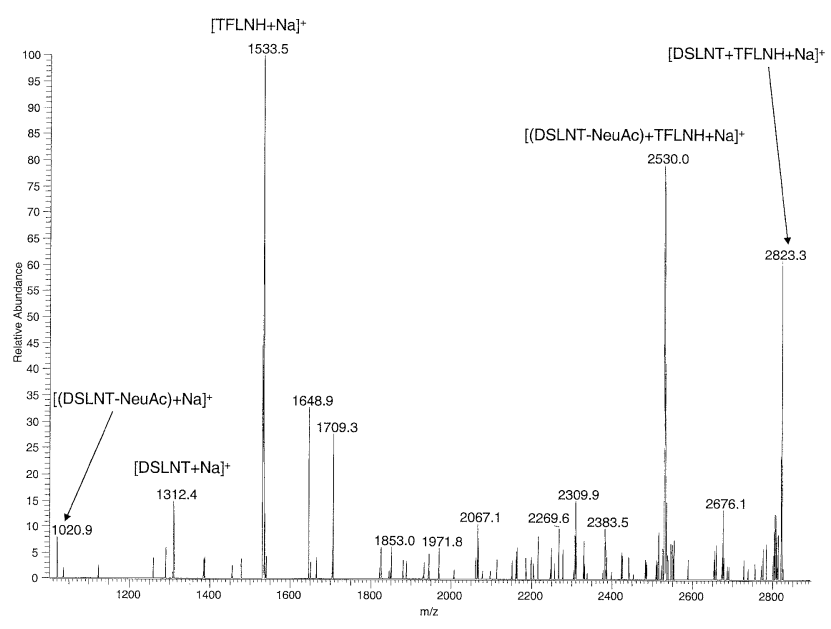

(b)

Figure 1. Positive mode CID spectra of sodiated disialyl-lactoN-tetraose (DSLNT) and trifucosyllacto-N-hexaose (TFLNH). (a) Full scan containing multiple cation attachments, (b) MS/MS of $[\mathrm{DSLNT}+\mathrm{TFLNH}+\mathrm{Na}]^{+}$at $\mathrm{m} / \mathrm{z}(2821.5)$.

trum, with the sodiated species predominating, due to the abundance of sodium present in glycerol. Disialyllacto-N-tetraose (DSLNT) (Scheme 1a) and trifucosyllacto-N-hexaose (TFLNH) were analyzed as a mixture. This figure shows sodiated homodimers of DSLNT $(\mathrm{m} / \mathrm{z}$ 2599.5) and trifucosyllacto-N-hexaose (3043.0) as well as the sodiated mixed dimer at $\mathrm{m} / \mathrm{z}$ 2821.5. These noncovalent complexes were presumed to be weak, due to the lack of potential salt-bridges (only one sugar contains ionizable groups). However, under CID conditions, the DSLNT-TFLNH noncovalent complex produced fragmentation indicative of tight association (Figure 1b). Fragmentation of the noncovalent complex results not only in the breakdown of the complex into its two substituents $(m / z 1312.4,1533.5)$, but also significant loss of sialic acid from the complex itself $(\mathrm{m} / \mathrm{z} 2253.0)$. This indicates that the noncovalent gas phase interactions between DSLNT and TFLNH can be stronger than the labile intramolecular bond of sialic acid. One possible cause for this increased affinity could be due to an extensive hydrogen bonding network between the two sugars. It was also hypothesized that the strong interactions were sialic acid dependent, with this sugar residue playing a key role in these noncovalent complexes.

Experiments were designed to test the range of interactions which lead to similar fragmentation. Mixtures of other sugars were analyzed to determine if these interactions were specific to sugars containing the lactose core (Gal $\beta 1-4 \mathrm{Glc})$, which both DSLNT and TFLNH contain. Oligomannose-3 (MAN3) (Scheme 1c) was chosen because of its different core structure (GlcNAc $\beta 1-4 \mathrm{GlcNAc}$ ) from that of DSLNT. Figure 2a shows the sodiated homodimers of DSLNT $(\mathrm{m} / z$ 2600.8) and MAN3 $(\mathrm{m} / \mathrm{z} 1842.3)$ as well as a sodiated trimer of MAN3 ( $m / z$ 2753.0). A noncovalent complex between DSLNT and MAN3 also appears in the spectrum at $\mathrm{m} / \mathrm{z}$ 2221.1. CID analysis of the DSLNT-MAN3 noncovalent complex demonstrates similar fragmentation to that of DSLNT-TFLNH (Figure 2b). The predominant fragments seen are the loss of sialic acid from the noncovalent complex $(\mathrm{m} / \mathrm{z}$ 1930.3) and the sodiated DSLNT $(\mathrm{m} / \mathrm{z}$ 1312.3). DSLNT has a higher affinity for sodium, which can be deduced from the lack of sodiated MAN3 in the fragmentation spectrum.

Analysis of the DSLNT-MAN3 mixture was also performed under negative mode ionization, to ensure strong noncovalent complex formation and affinity was not due to the presence of sodium (Figure 2c, d). The full scan analysis of the mixture shows the noncovalent complex between DSLNT and MAN3 ( $\mathrm{m} / \mathrm{z} 2196.9)$, as well as the complex with loss of sialic acid before CID $(\mathrm{m} / \mathrm{z}$ 1906.0) (Figure 2c). Figure $2 \mathrm{~d}$ is the negative mode CID analysis of DSLNT-MAN3 complex. The predominant peak is the loss of sialic acid $(\mathrm{m} / \mathrm{z}$ 1906.0), which again indicates that the strength of the noncovalent complex is stronger than the covalent bond linkage to sialic acid. These results are similar to results seen with the sodiated complex. An additional fragment ion $(\mathrm{m} / \mathrm{z}$ 2017.9) resulting from the loss of 180 could be the result of the $\mathrm{B}_{4}$ cleavage of DSLNT, according to fragmentation nomenclature described by Domon and Costello [28]. This would indicate stronger affinity under negative mode analysis since this is not a labile bond.

Additional analyses were performed to elucidate the structures involved in determining binding strength. Two sugars containing sialic acids were mixed DSLNT and disialyl,monofucosyllacto-N-hexaose (DSFLNH) (Scheme 1d). The negative mode full scan analysis of DSLNT and DSFLNH shows both homodimers of DSLNT $(\mathrm{m} / \mathrm{z}$ 2576.1) and DSFLNH (3598.9) and heterodimers between the two sugars $(\mathrm{m} / \mathrm{z}$ 3088.1) (Figure 3a). CID analysis again depicts the loss of sialic acid from the noncovalent complex $(\mathrm{m} / \mathrm{z} 2797.0)$, without significant breakdown into individual substituents (Figure $3 b)$. Peaks representing multiple losses of sialic acid are also present in the spectrum $(m / z 2506.5)$, as well as 


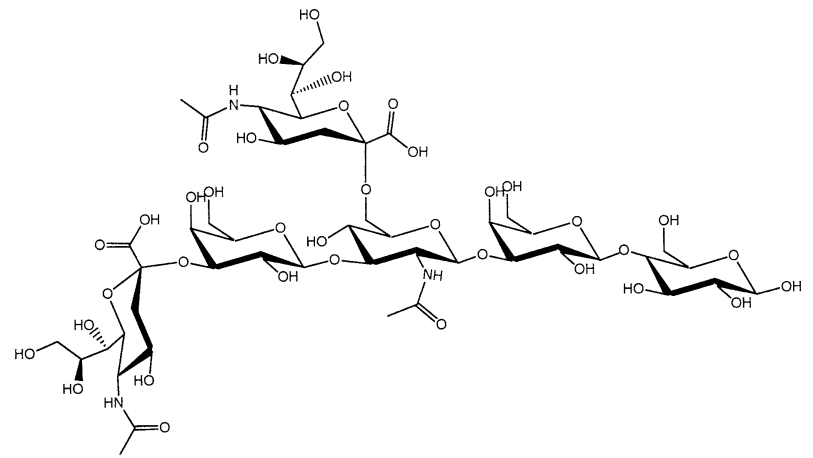

(a)

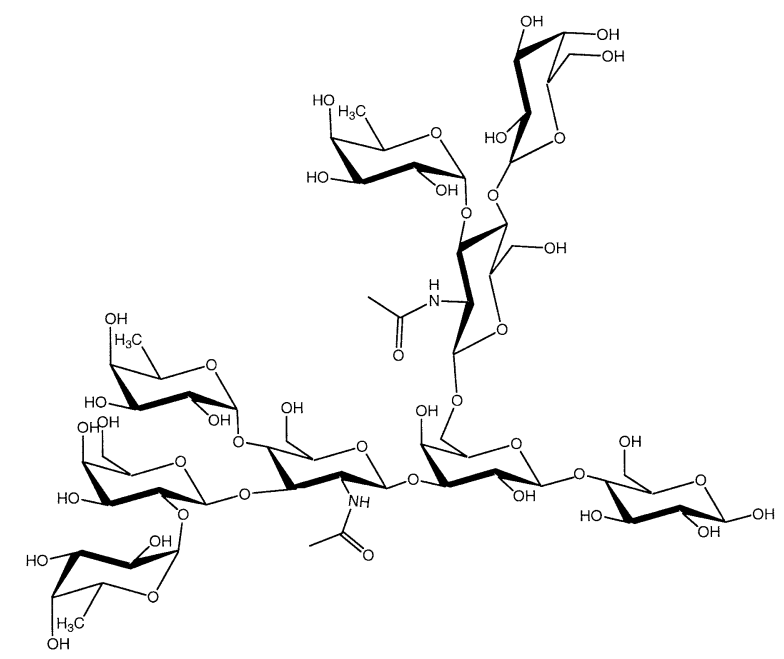

(b)
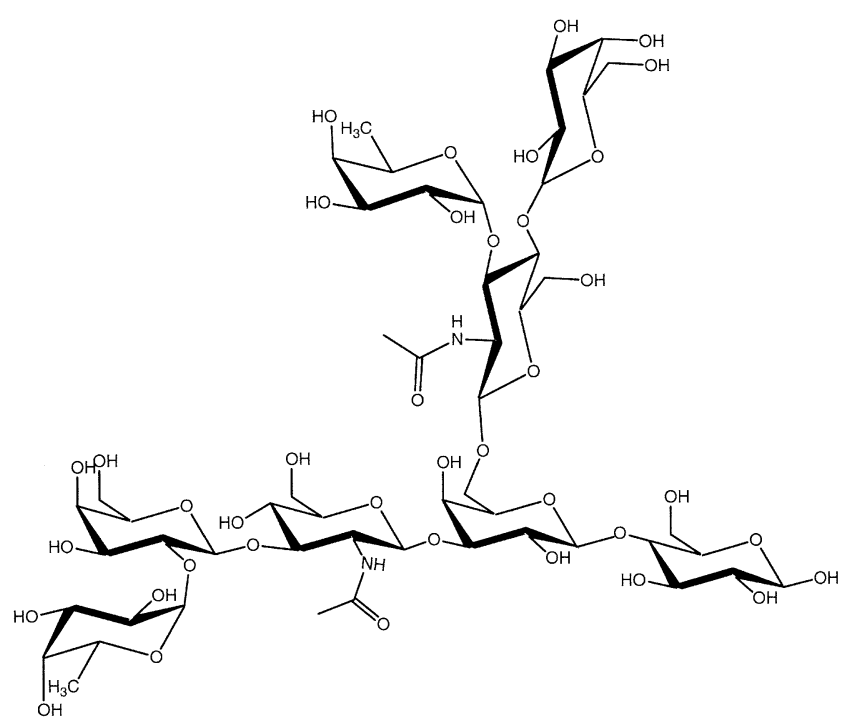

(e)

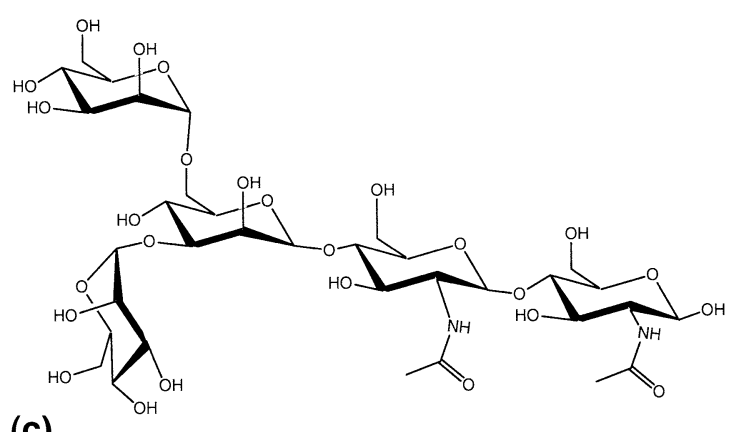

(c)

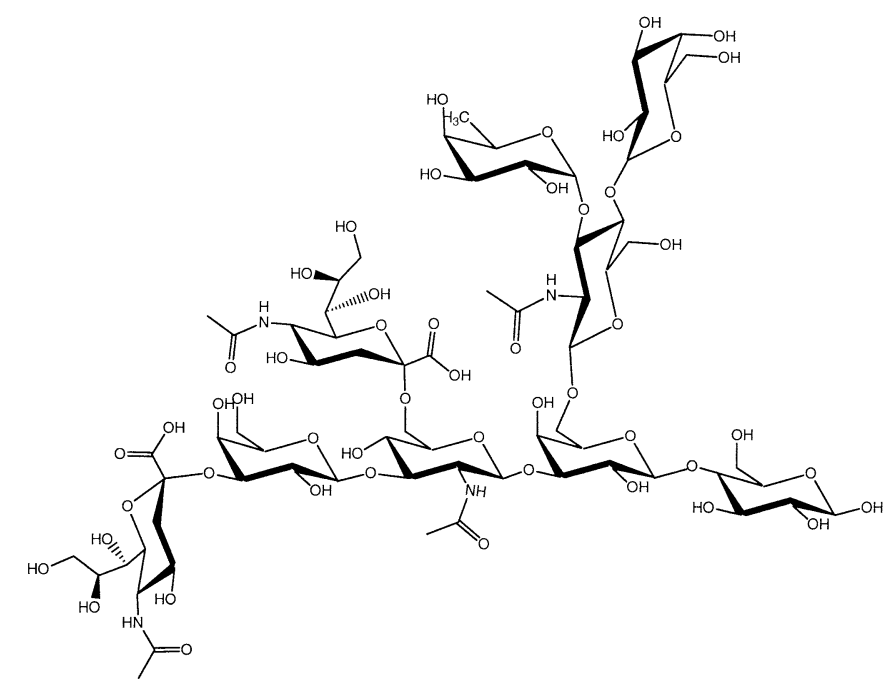

(d)
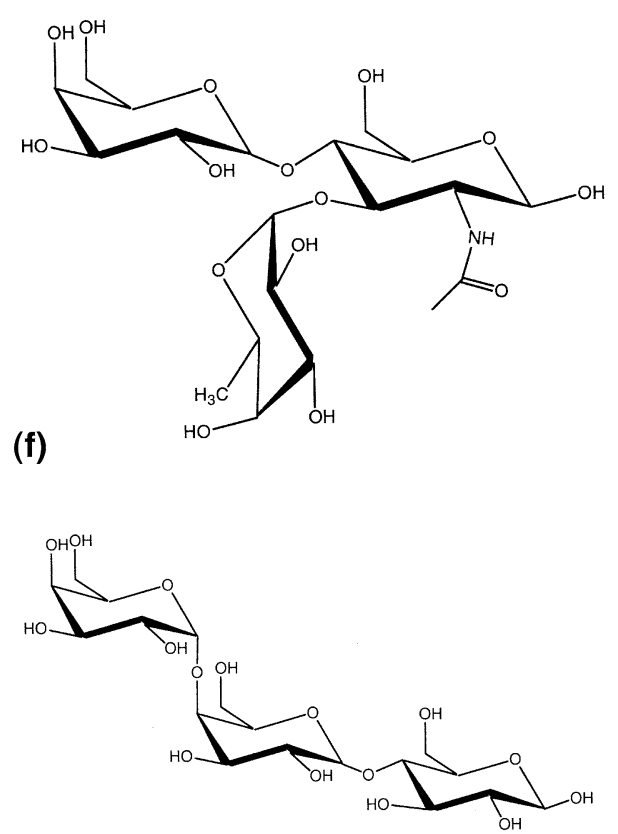

(g)

Scheme 1. Structures of (a) disialyl-lacto-N-tetraose, (b) trifucosyllacto-N-hexaose, (c) oligomannose-3, (d) disialyl,monofucosyllacto-N-hexaose, (e) difucosyllacto-N-hexaose(a), (f) Lewis ${ }^{\mathrm{X}},(\mathrm{g})$ globotrios. 


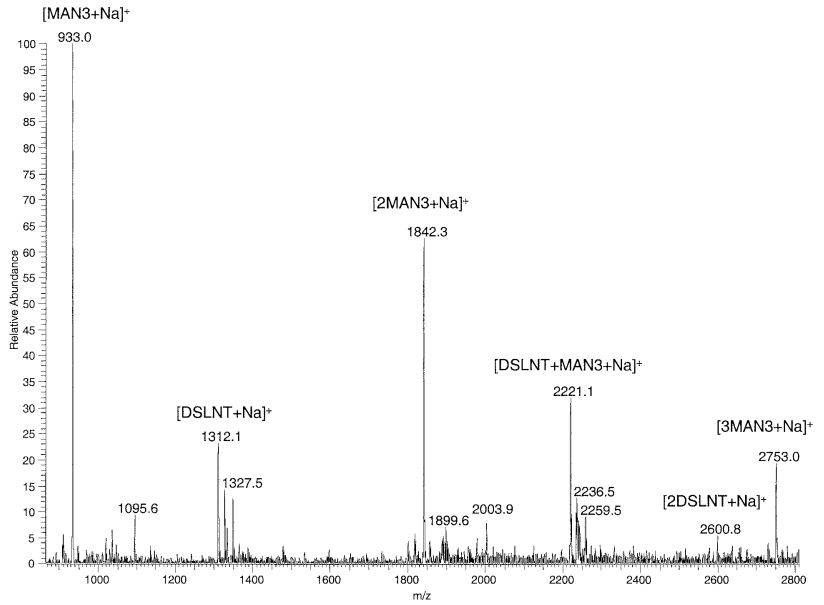

(a)

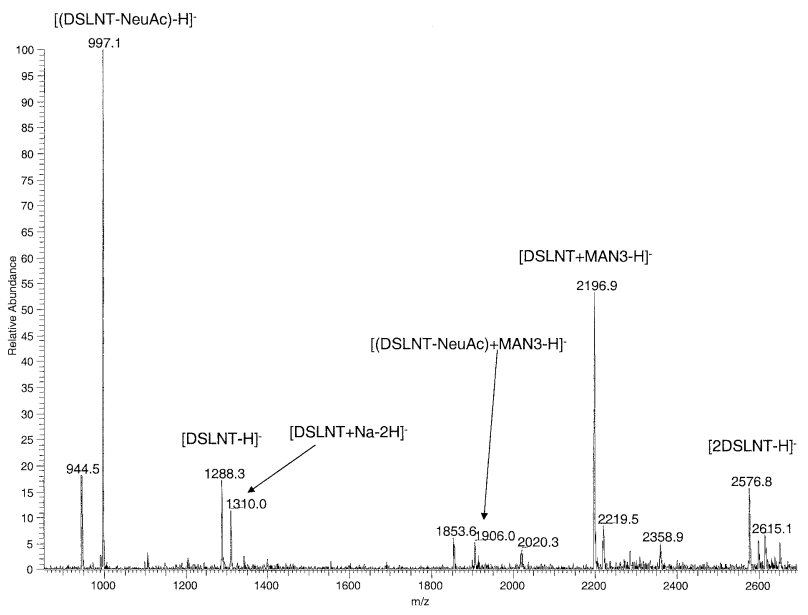

(c)

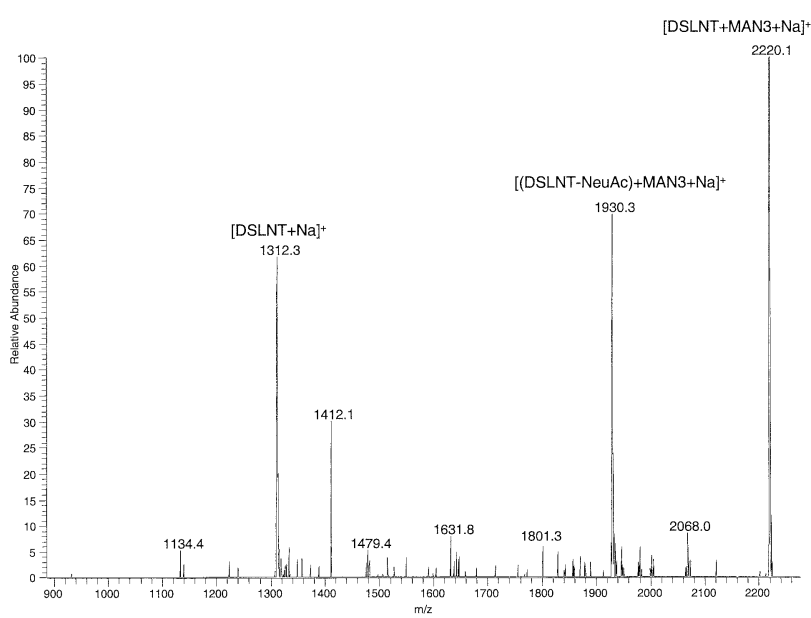

(b)

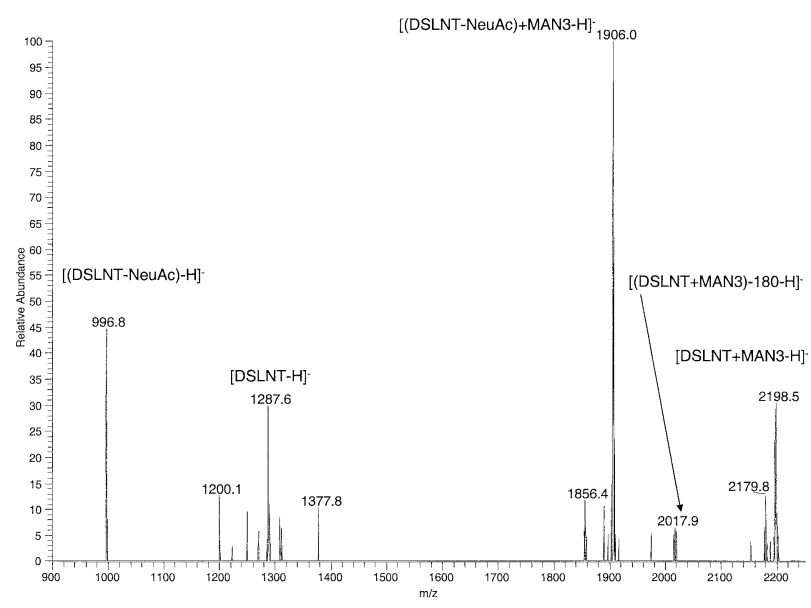

(d)

Figure 2. CID spectra of disialyl-lacto-N-tetraose (DSLNT) and oligomannose-3 (MAN3). (a) Positive mode full scan, (b) positive mode MS/MS of [DSLNT + MAN3 + Na] ${ }^{+}$at $m / z(2221.1)$, (c) negative mode full scan, (d) negative mode MS/MS of [DSLNT + MAN3 - H] ${ }^{-}$at $m / z(2196.9)$.

a peak resulting from the addition of a sialic acid residue to DSFLNH $(\mathrm{m} / \mathrm{z} 2093.5)$. The presence of $\mathrm{m} / \mathrm{z}$ 2093.5 indicates that sialic acid is a mediator in complex formation, and can associate tightly with compounds in close proximity.

Complexes between two neutral sugars were studied in order to test whether sialic acid was required for strong binding. Oligomannose-3 was mixed with difucosyllacto-N-hexaose(a) (DFLNHa), both of which lack sialic acid and are uncharged at physiological $\mathrm{pH}$. DFLNHa contains multiple fucose residues, known to be labile under mass spectrometric analysis (Scheme 1e). The full scan analysis of this mixture shows sodiated homodimers and homotrimers of MAN3 $(\mathrm{m} / \mathrm{z}$ $1842.0,2750.1)$ ) as well as the noncovalent complex between MAN3 and DFLNHa ( $m / z$ 2295.8) (Figure 4a). CID analysis of the sodiated noncovalent complex results in little fragmentation beyond the complex breakdown. The predominant peaks represent the sugar monomers (MAN3 m/z 932.8 and DFLNHa $m / z$ 1387.3).
Consequently, fragmentation of sugar-sugar complexes does not always result in loss of labile groups. Different noncovalent complexes have different binding affinities, which accounts for the differences in fragmentation patterns. Tighter complexes can result in preferential loss of covalent bonds before destruction of the complex itself.

Attention was then turned to sugars reported to be involved in cell-cell interactions. Sugars have been demonstrated to be the key residues involved in some forms of cellular interactions. This technique could aid in the study of these noncovalent interactions. Two sugars were chosen because of their biological significance, Lewis ${ }^{x}$ and globotriose (Scheme 1f and $\mathbf{g}$ ). Lewis ${ }^{x}$ has been reported to interact tightly with itself, and is known to be a binding determinant for some cellular processes [12]. Globotriose is the core structure of the carbohydrate domain of GSLs known to play a role in cell-cell interactions mediated exclusively by sugars [8].

Lewis $^{\mathrm{x}}$-Lewis ${ }^{\mathrm{x}}$ noncovalent complex formation was 


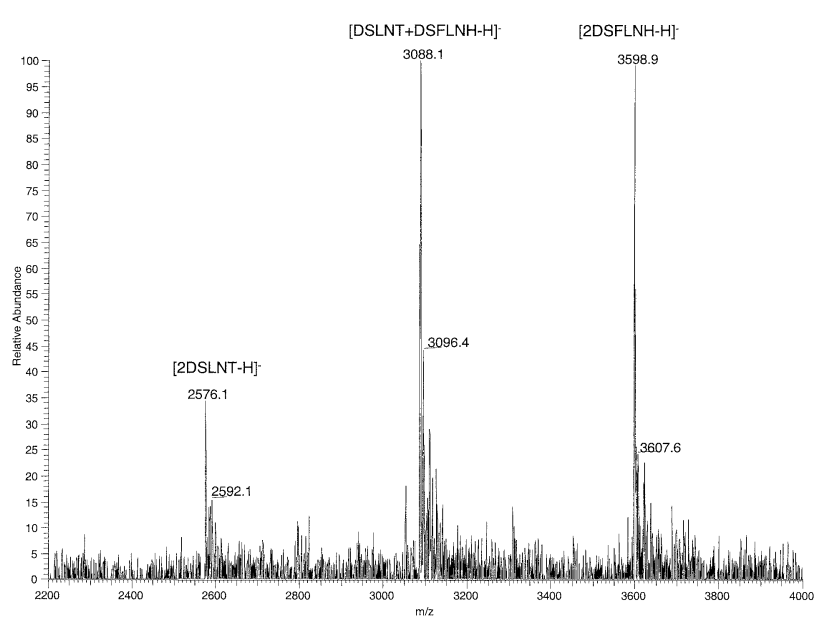

(a)

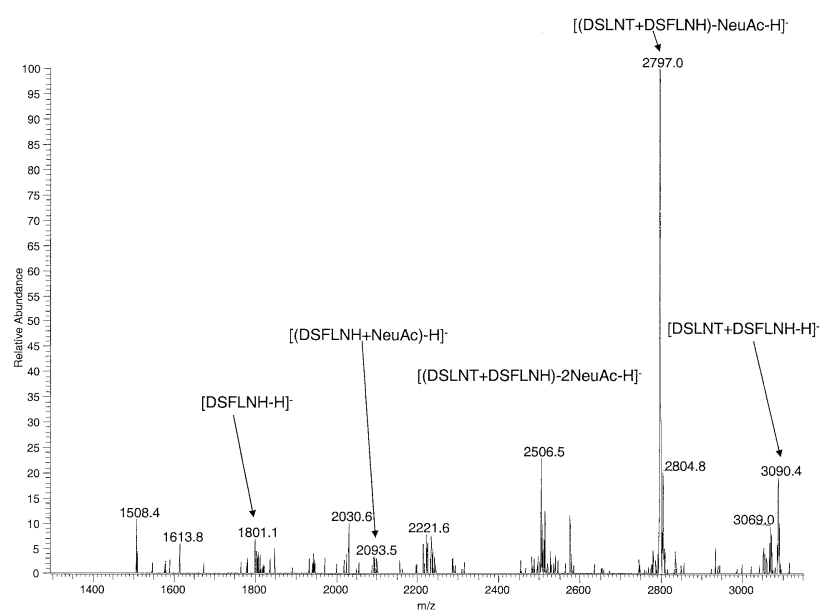

(b)

Figure 3. Negative mode CID spectra of disialyl-lacto-N-tetraose (DSLNT) and disialyl,monofucosyllacto-N-hexaose (DSFLNH). (a) Full scan, (b) MS/MS of [DSLNT + DSFLNH - H] ${ }^{-}$at $\mathrm{m} / \mathrm{z}$ (3088.1).

analyzed in positive mode (Figure 5). The full scan spectrum shows the presence of both sodiated monomer $(m / z 552.3)$ and sodiated dimer $(m / z$ 1081.0) forms of Lewis ${ }^{x}$ (Figure 5a). CID analysis of the noncovalent complex shows breakdown of the complex into monomers, with no significant fragmentation (Figure 5b). This result is similar to that seen with the complex between MAN3 and DFLNHa.

Globotriose was also analyzed to determine its dimer fragmentation pattern. The full scan analysis illustrates complex formation $(\mathrm{m} / \mathrm{z}$ 1031.0) (Figure 6a). CID analysis of the globotriose-globotriose noncovalent complex shows significant fragmentation before complex breakdown, unlike that of the Lewis ${ }^{\mathrm{x}}-$ Lewis $^{\mathrm{x}}$ dimers (Figure $6 \mathrm{~b})$. No globotriose monomer is present in the CID spectrum. Fragmentation indicates rearrangement between the dimers, which indicates very tight binding of the sodiated globotriose noncovalent complex. This result indicates that fragmentation is not completely

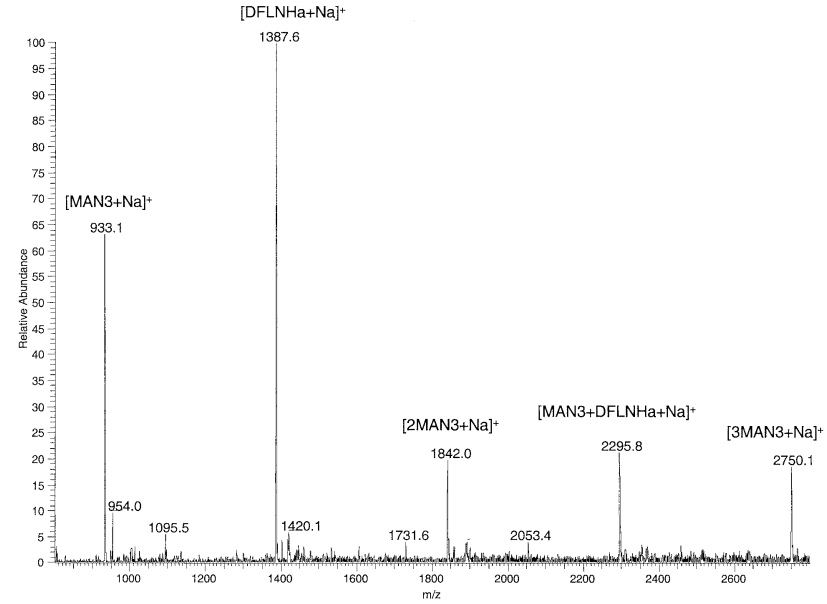

(a)

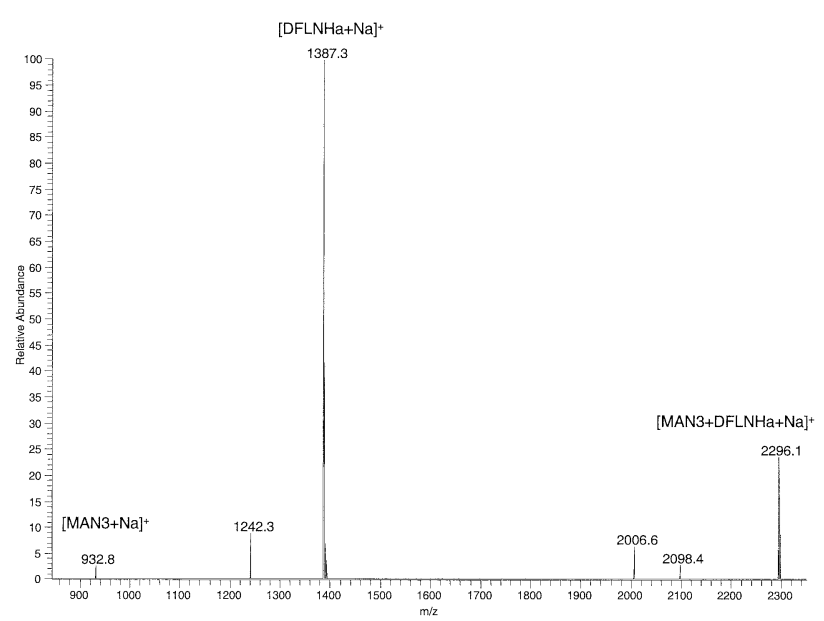

(b)

Figure 4. Positive mode CID spectra of sodiated oligomannose-3 (MAN3) and difucosyllacto-N-hexaose(a) (DFLNHa). (a) Full scan, (b) MS/MS of [MAN3 + DFLNHa $+\mathrm{Na}]^{+}$at $\mathrm{m} / \mathrm{z}(2295.8)$.

sialic acid dependent, and that neutral species can form extensive hydrogen bonding networks.

Sugar-sugar noncovalent complexes have higher binding affinities under gas phase conditions than we might have expected. The fragmentation of intramolecular bonds prior to complex breakdown indicates the high affinity of the noncovalent interactions. These interactions are most likely due to a series of hydrogen bonds. A single hydrogen bond is a relatively weak interaction. Several hydrogen bonds between various sugar residues could account for the strength of interactions. A series of hydrogen bonds could also account for differences in complex strength and formation between sugars of different structures. Proper alignment would be needed to form the strongest interactions, and sugars with varying positions of hydrogen bonding donors and acceptors could vary the resulting interaction.

Noncovalent complex formation and fragmentation can occur in both positive and negative mode mass 


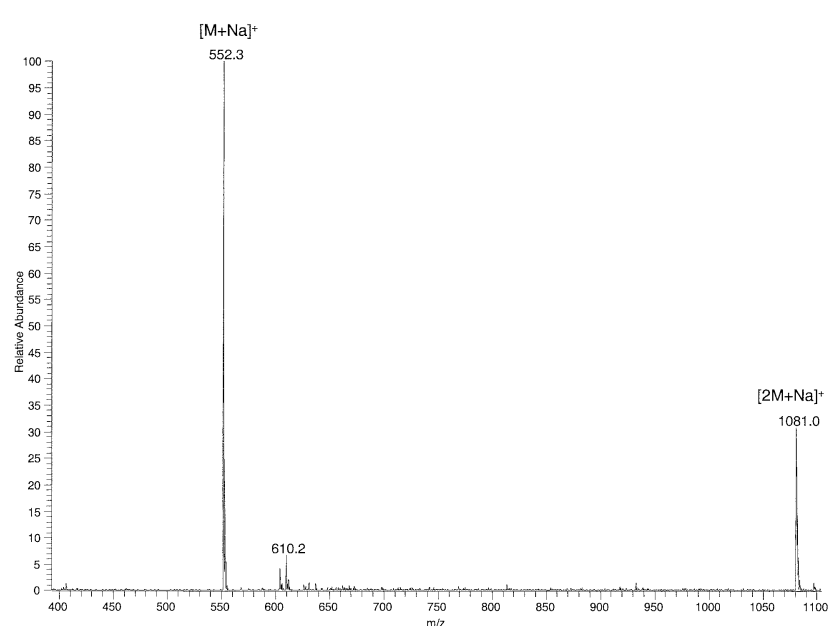

(a)

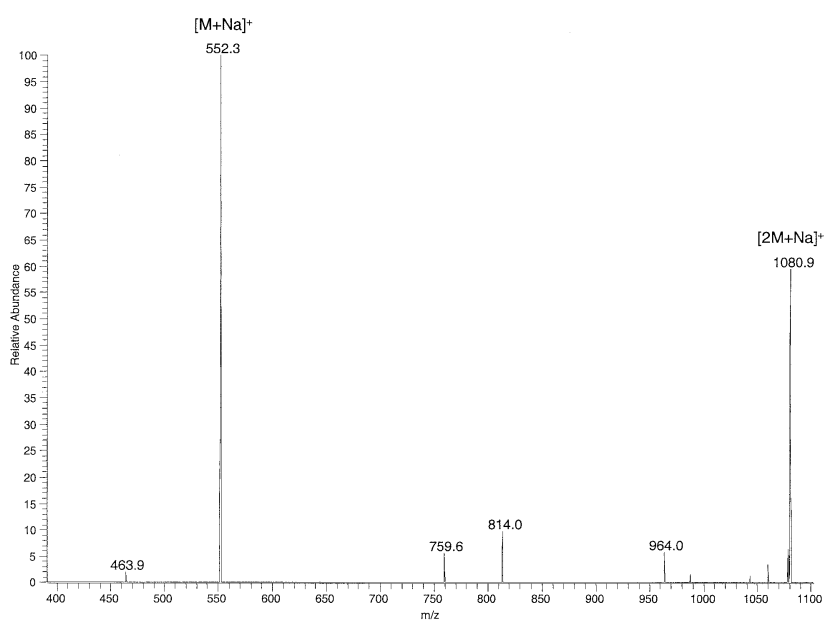

(b)

Figure 5. Positive mode CID spectra of sodiated Lewis ${ }^{\mathrm{x}}$ dimer. (a) Full scan, (b) MS/MS of $[2 \mathrm{M}+\mathrm{Na}]^{+}$at $m / z(1081)$.

spectrometric analysis. As such, noncovalent complex formation does not appear to be the result of a cationbound dimer. Furthermore, a larger variety of sugars can be studied, since some sugars ionize more readily under negative mode than positive mode. Since both positive and negative mode produce similar fragmentation patterns (Figure 2), fragmentation of complexes under different conditions can be compared.

Charge also appears to play a role in the strength of interaction between noncovalent sugar-sugar complexes. Sugars containing sialic acid appear to form strong dimers, exclusively breaking intramolecular bonds before the breakdown of the complex. This could be due to formation of extremely strong interactions within the core of sugars containing sialic acids. It could also be a result of activation of cleavage of the already labile sialic acid bond through limiting the rotational and vibrational freedom of the sialylated sugar while in the noncovalent complex. The result is a noncovalent complex with a strength of interaction higher than the sialic acid linkage.

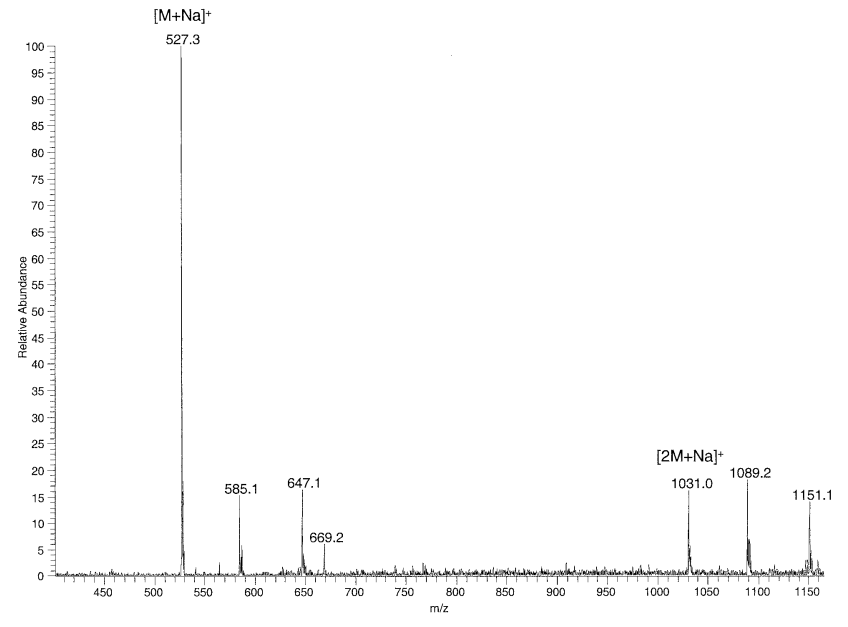

(a)

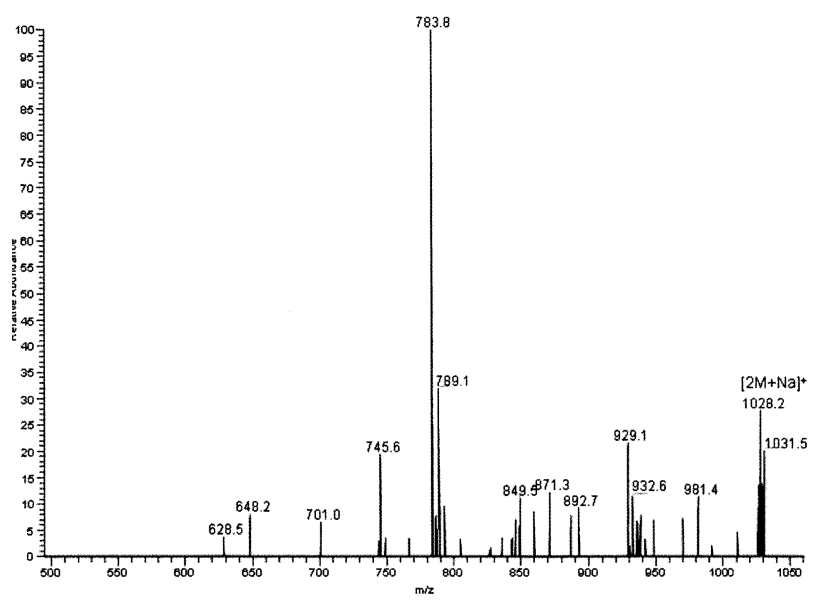

(b)

Figure 6. Positive mode CID spectra of sodiated globotrios dimer. (a) Full scan, (b) MS/MS of $[2 \mathrm{M}+\mathrm{Na}]^{+}$at $\mathrm{m} / \mathrm{z}(1031.0)$.

This technique can be used to study sugars with known involvement in intercellular interactions. Globotriose is the core portion of the sugar on glycosphingolipids known to interact leading to downstream cellular effects [8]. Noncovalent dimers of globotriose have strong interactions, which result in sugar fragmentation and rearrangement before complex breakdown. This indicates a high strength of interaction, which in itself could lead to downstream cellular effects, without the need for protein binding. This finding could lead to further searches for sugars mediating cellular interactions, and could greatly aid the field of glycobiology.

\section{Conclusions}

IR AP MALDI produces thermally cooled ions, which allows the study of noncovalent complexes. Weak complexes can be maintained during ionization, due to the "soft" ionization process, as well as the near physiological conditions of the glycerol matrix. Once formed, 
sugar-sugar noncovalent complexes show varying strength and affinity, with markedly different fragmentation patterns. Noncovalent complex formation and fragmentation is sugar specific, with different sugars having different affinities, and fragmentation patterns. Fragmentation of complexes containing sugars with sialic acids produce spectra that retain the complex with the loss of sialic acid, rather than the expected breakdown of the noncovalent complex itself. Neutral sugars do not seem to undergo intramolecular fragmentation, rather they seem to break down into the monomer substituents that form the complex. One biologically relevant complex resulting from globotriose dimers does, however, show significant fragmentation before breakdown of the noncovalent complex. Future work will attempt to identify key residues involved in complex formation, as well as additional tests to determine strength of interaction. Methods to disrupt hydrogen bond formation could eliminate complex formation or sugar-sugar affinity, and will provide additional insights into complex specificity. In addition, the ability to control and alter energy and excitation time in the ion trap may be used to characterize the relative binding strength of the complexes. At this time, however, the results demonstrate the effectiveness and value of using IR AP MALDI for the study of sugar-sugar noncovalent interactions. Using this technique, interactions involving other sugars can be tested for their roles in cellular interactions.

\section{Acknowledgments}

Funding for this work is provided by a contract (DABT63-99-10006) to RJC from the Defense Advanced Research Project Agency (DARPA). CEV is supported by an NIH Training Grant in AntiCancer Drug Development (CA 09243).

\section{References}

1. Varki, A. Sialic Acids as Ligands in Recognition Phenomena. FASEB J. 1997, 11, 248-255.

2. Varki, A. Biological Roles of Oligosaccharides: All of the Theories are Correct. Glycobiology 1993, 3, 97-130.

3. Crocker, P. R.; Varki, A. Siglecs in the Immune System. Immunology 2001, 103, 137-145.

4. Schauer, R. Achievements and Challenges of Sialic Acid Research. Glycoconj. J. 2000, 17, 485-499.

5. Kelm, S.; Schauer, R. Sialic Acids in Molecular and Cellular Interactions. Int. Rev. Cytol. 1997, 175, 137-240.

6. Hakomori, S. The Glycosynapse. PNAS 2002, 99, 225-232.

7. Handa, K.; Kojima, N.; Hakomori, S. Analysis of GlycolipidDependent Cell Adhesion Based on Carbohydrate-Carbohydrate Interaction. Methods Enzymol. 2000, 312, 447-458.

8. Hakomori, S. Structure, Organization, and Function of Glycosphingolipids in Membrane. Current Opin. Hematol. 2003, 10, $16-24$.

9. Hakomori, S. Cell Adhesion/Recognition and Signal Transduction Through Glycosphingolipid Microdomain. Glycoconj. J. 2000, 17, 143-151.

10. Gege, C.; Geyer, A.; Schmidt, R. R. Carbohydrate-Carbohydrate Recognition Between Lewis X Blood Group Antigens, Mediated by Calcium Ions. Eur. J. Org. Chem. 2002, 2475, 2485.
11. Hakomori, S. Traveling the Glycosphingolipid Path. Glycoconj. J. 2000, 17, 627-647.

12. Loo, J. A. Electrospray Ionization Mass Spectrometry: A Technology for Studying Noncovalent Macromolecular Complexes. Int. J. Mass Spectrom. 2000, 200, 175-186.

13. Veenstra, T. D. Electrospray Ionization Mass Spectrometry in the Study of Bimolecular Noncovalent Interactions. Biophys. Chem. 1999, 79, 63-79.

14. Pramanik, B. N.; Bartner, P. L.; Mirza, U. A.; Liu, Y.; Ganguly, A.K. Electrospray Ionization Mass Spectrometry for the Study of Noncovalent Complexes: An Emerging Technology. J. Mass Spectrom. 1998, 31, 911-920.

15. Woods, A. S.; Koomen, J. M.; Ruotolo, B. T.; Gillig, K. J.; Russel, D. H.; Fuhrer, K.; Gonin, M.; Egan, T. F.; Schultz, J. A. A Study of Peptide-Peptide Interactions Using MALDI Ion Mobility o-TOF and ESI Mass Spectrometry. J. Am. Soc. Mass Spectrom. 2002, 13, 166-169.

16. Lin, S.; Long, S.; Ramirez, S. M.; Cotter, R.J.; Woods, A. S. Characterization of the "Helix Clamp" Motif of HIV-1 Reverse Transcriptase Using MALDI-TOF and Surface Plasmon Resonance. Anal. Chem. 2000, 72, 2635-2640.

17. Jespersen, S.; Niessen, W. M. A.; Tjaden, U. R.; van der Greef, J. Basic Matrices in the Analysis of Noncovalent Complexes by Matrix-Assisted Laser Desorption/Ionization Mass Spectrometry. J. Mass Spectrom. 1998, 33, 1088-1093.

18. Laiko, V. V.; Baldwin, M. A.; Burlingame, A. L. Atmospheric Pressure Matrix-Assisted Laser Desorption/Ionization Mass Spectrometry. Anal. Chem. 2000, 72, 652-7.

19. Laiko, V. V.; Moyer, S. C.; Cotter, R. J. Atmospheric Pressure MALDI/Ion Trap Mass Spectrometry. Anal. Chem. 2000, 72, 5239-5243.

20. Moyer, S. C.; Marzilli, L. A.; Woods, A. S.; Laiko, V. V.; Doroshenko, V. M.; Cotter, R. J. Atmospheric Pressure Matrix Assisted-Laser Desorption/Ionization (AP MALDI) on a Quadrupole Ion Trap Mass Spectrometer. Int. J. Mass Spectrom. 2003, 226, 133-150.

21. Spengler, B.; Kirsch, D.; Kaufmann, R. Fundamental Aspects of Postsource Decay in Matrix-Assisted Laser Desorption Mass Spectrometry. 1. Residual Gas Effects. J. Phys. Chem. 1992, 96, 9678-9684.

22. Creaser, C. S.; Reynolds, J. C.; Harvey, D. J. Structural Analysis of Oligosaccharides by Atmospheric Pressure Matrix-Assisted Laser Desorption/Ionization Quadrupole Ion Trap Mass Spectrometry. Rapid Commun. Mass Spectrom. 2002, 16, 176-184.

23. Danell, R. M.; Glish, G. L. An Atmospheric Pressure MALDI Probe for Use with ESI Source Interfaces, Proceedings of the 48th ASMS Conference on Mass Spectrometry and Allied Topics; Long Beach, CA, June, 2000.

24. Miller, C. A.; Yi, D.; Perkins, P. D. An Atmospheric Pressure Matrix-Assisted Laser Desorption/Ionization Ion Trap with Enhanced Sensitivity. Rapid Commun. Mass Spectrom. 2003, 17, 860-868.

25. Galicia, M. C.; Vertes, A.; Callahan, J. H. Atmospheric Pressure Matrix-Assisted Laser Desorption/Ionization in Transmission Geometry. Anal. Chem. 2002, 74, 1891-1895.

26. Laiko, V. V.; Taranenko, N. I.; Berkout, V. D.; Yakshin, M. A.; Prasad, C. R.; Lee, H. S.; Doroshenko, V. M. Desorption/ Ionization of Biomolecules from Aqueous Solutions at Atmospheric Pressure Using an Infrared Laser at $3 \mu \mathrm{m}$. J. Am. Soc. Mass Spectrom. 2002, 13, 354-361.

27. Von Seggern, C. E. Moyer, S. C. Cotter, R. J. Liquid Infrared Atmospheric Pressure MALDI/Ion Trap Mass Spectrometry of Sialylated Carbohydrates, Anal Chem., 2003, 75, 3212-3218.

28. Domon, B.; Costello, C. A Systematic Nomenclature for Carbohydrate Fragmentations in FAB-MS/MS Spectra of Glycoconjugates. Glycoconj. J. 1988, 5, 397-409. 